\title{
Endarterectomy for symptomatic non- stenotic carotids: a systematic review and descriptive analysis
}

\author{
Anthony Larson (D) , ${ }^{1}$ Valentina Nardi, ${ }^{1,2}$ Waleed Brinjikji, ${ }^{1,2}$ John Benson, ${ }^{1}$ \\ Giuseppe Lanzino, ${ }^{1,2}$ Luis Savastano ${ }^{2}$
}

To cite: Larson A, Nardi V, Brinjikji W, et al. Endarterectomy for symptomatic non-stenotic carotids: a systematic review and descriptive analysis. Stroke \& Vascular Neurology 2021;0. doi:10.1136/svn-2021-001122

- Additional supplemental material is published online only. To view, please visit the journal online (http://dx.doi.org/10. 1136/svn-2021-001122).

Received 20 May 2021 Accepted 22 June 2021
Check for updates

(C) Author(s) (or their employer(s)) 2021. Re-use permitted under CC BY-NC. No commercial re-use. See rights and permissions. Published by BMJ.

${ }^{1}$ Department of Radiology, Mayo Clinic, Rochester, Minnesota, USA

${ }^{2}$ Neurosurgery, Mayo Clinic, Rochester, Minnesota, USA

Correspondence to Dr Luis Savastano;

Savastano.luis@mayo.edu

\section{ABSTRACT}

Objective To systematically analyse prior reports of carotid endarterectomy (CEA) performed in cases of $\leq 50 \%$ carotid stenosis in order to understand patient tolerance and potential benefit.

Methods A systematic review and descriptive analysis was performed in concordance with the Preferred Reporting Items for Systematic Reviews and MetaAnalyses guidelines. An English-language search was performed of online databases using librarian-selected search terms. Abstracts were reviewed for relevance which included mention of carotid endarterectomy and stenosis. Prospective or retrospective observational cohort studies that reported series of patients who underwent endarterectomy for minimal $(\leq 50 \%)$ luminal stenosis with reported outcomes were included.

Results Six studies (which included our institutional series) with a total of 143 patients met the inclusion criteria. The weighted mean age at the time of CEA was 72.3 years; $113(79.0 \%)$ were male. $55.8 \%$ of patients with available data had recurrent ipsilateral ischaemic events despite medical therapy. Two patients out of 129 with available perioperative data (1.6\%) had perioperative MRI findings of acute ischaemic stroke, both within the hemisphere contralateral to the side of CEA. Of the 138 patients with available follow-up (mean, 36 months), none had recurrent ipsilateral ischaemic events.

Conclusions Endarterectomy for symptomatic carotid disease causing $\leq 50 \%$ stenosis may be a potentially beneficial strategy to prevent stroke recurrence. Studies with robust methodology are needed to draw more definitive conclusions in terms of the safety and efficacy of endarterectomy for minimal stenosis with vulnerable features relative to intensive medical therapy.

\section{INTRODUCTION}

Atherosclerotic disease of the carotid arteries carries a well-known association with ipsilateral cerebral ischaemic events, including stroke. As a result of landmark trials including the North American Symptomatic Carotid Endarterectomy Trial (NASCET), ${ }^{1}$ decisions regarding invasive treatment have been based primarily on the degree of carotid luminal stenosis. ${ }^{2}$ Indeed, the current guidelines reflect the central role of the degree of stenosis in determining whether intervention is warranted. ${ }^{34}$ However, a growing body of evidence suggests that not all symptomatic plaques result in clinically significant stenosis. Instead, symptomatic plaques are often found to contain histologically 'vulnerable' features that make them susceptible to thrombosis and distal embolisation. ${ }^{56}$

Recent advances in imaging have allowed for greater non-invasive characterisation of plaque features, such as intraplaque haemorrhage (IPH) ${ }^{6-8}$ As use of these imaging techniques has increased, so too has the identification of patients with mildly stenotic yet vulnerable plaques that are thought to be the culprit lesions in some embolic strokes of undetermined source (ESUS)..${ }^{5910}$ As such, the presence of vulnerable or 'unstable' features of carotid plaques is likely an important imaging biomarker in considering interventional measures, even in cases of minimal stenosis.

Recently, much attention has been given to strokes in the context of minimal ipsilateral carotid stenosis with vulnerable features. Goyal $e t a l^{11}$ have proposed symptomatic nonstenotic carotid artery disease (SyNC) as a working definition, which may be a more appropriate classification schema for patients with stroke in the context of minimal ipsilateral stenosis and vulnerable plaque features. With this definition, patients with SyNC may be candidates for targeted treatment, which is in contrast to the same patients who, in the absence of $>50 \%$ ipsilateral carotid stenosis or another source of stroke, are classified as having an ESUS. Accordingly, ESUS precludes treatment with targeted therapy.

It is reasonable to hypothesise that patients meeting the proposed criteria for SyNC may benefit from the targeted therapy of carotid endarterectomy (CEA). To date, however, few studies have reported the safety and efficacy of surgical intervention on symptomatic carotid plaques with minimal $(\leq 50 \%)$ stenosis, let alone in the context of SyNC as proposed by Goyal et al. ${ }^{11} \mathrm{~A}$ logical first step in determining if CEA may be an effective targeted therapy 
in cases of SyNC is determining the safety and efficacy of CEA in the context of minimal stenosis. In the absence of more robust data, an understanding of the current data that exist in the literature is an important step in establishing the role of surgical intervention on carotid plaques with minimal stenosis, as well as guiding future studies. The purpose of the current study was therefore to perform a systematic review and descriptive analysis of all published reports of surgical intervention on symptomatic carotid atherosclerotic plaques with minimal stenosis.

\section{METHODS}

\section{Search strategy and selection criteria}

This study was a systematic review and descriptive analysis that was performed according to the Preferred Reporting Items for Systematic Reviews and Meta-Analyses. ${ }^{12} \mathrm{~A}$ comprehensive search of several databases from inception to 1 June 2020, limited to English language and excluding animal studies, was conducted. The databases included Ovid MEDLINE(R) and Epub Ahead of Print, In-Process and Other Non-Indexed Citations and Daily, Ovid Embase, Ovid Cochrane Central Register of Controlled Trials, Ovid Cochrane Database of Systematic Reviews, and Scopus. The search strategy was designed and conducted by an experienced librarian with input from the study's principal investigator. Controlled vocabulary supplemented with keywords was used to search for studies describing CEA in cases of symptomatic patients with less than $50 \%$ stenosis. The actual strategy listing all search terms used and how they are combined is available in the online supplemental appendix.

Abstracts were reviewed for relevance which included mention of carotid endarterectomy and stenosis. Abstracts without mention of both of these variables were excluded and the full text was not reviewed. A study was considered eligible for this meta-analysis if it fulfilled the predefined inclusion criteria: prospective or retrospective observational cohort studies (single-arm or with a control group) that reported series of patients who underwent endarterectomy for minimal $(\leq 50 \%)$ luminal stenosis with reported outcomes were included.

\section{Data extraction and definitions}

From each included study, data were collected including first author, year of publication, number of patients, age, sex, imaging features, perioperative (within 30 days of CEA) complications, follow-up length and presence of major recurrent ischaemic events, and carotid artery restenosis at follow-up. Perioperative complications were classified as minor (haematoma, transient cranial nerve palsy, incisional bleeding) and major (permanent neurological deficit or ischaemic stroke as evidenced by MRI). Major recurrent ischaemic events were defined as permanent neurological deficits or an ischaemic stroke as evidenced by MRI.

Imaging features that were collected included the modality used, degree of luminal stenosis and presence of carotid IPH. Luminal stenosis was determined based on the NASCET criteria. ${ }^{1}$ The risk of bias assessment was performed with the Robins-I tool for non-randomised studies. ${ }^{13}$

\section{Subgroup analysis}

In order to understand the safety and efficacy of CEA on symptomatic plaques with $\leq 50 \%$ stenosis in the context of IPH, we performed a subgroup analysis of patients with carotid IPH as evidenced by magnetic resonance angiography (MRA) imaging studies who underwent subsequent endarterectomy. All patients who had MRA evidence of IPH at any time point preoperatively were included in this subgroup analysis.

\section{Statistical analysis}

The primary aims of this study were to (1) ascertain the rates of perioperative complications (as defined above) and (2) determine the prevalence of major recurrent ischaemic events at follow-up (as defined above) in patients who underwent CEA for symptomatic carotid stenosis of $\leq 50 \%$. Percentages were calculated for categorical variables including sex, rates of perioperative complications and long-term outcome results. Mean and SD were calculated for continuous variables including age and follow-up time. Weighted means were calculated in cases where means were reported in each individual study (ie, degree of ipsilateral stenosis, follow-up time). The descriptive nature of this review and descriptive analysis precluded the use of any formal statistical testing. All calculations took place in Microsoft Excel.

\section{RESULTS}

\section{Search results}

One hundred and forty-one abstracts were reviewed. There were nine full-text studies that were reviewed. Three full-text articles were excluded for various reasons: one study included patients with less than $50 \%$ stenosis that were treated with medical therapy alone ${ }^{14}$ and two studies included patients with degree of carotid stenosis greater than $50 \%{ }^{15}{ }^{16}$ Six studies met the inclusion criteria and were included in the final analysis. ${ }^{17-22}$ Study characteristics are summarised in table 1. Our search strategy is outlined in figure 1. All studies were found to have a moderate risk of bias by Robins-I tool (online supplemental table 1).

\section{Patient characteristics and outcomes}

In total, 143 patients underwent CEA for symptomatic carotid stenosis $\leq 50 \%$. One hundred and thirteen patients $(79.0 \%)$ were male. The weighted mean age at the time of CEA was 72.3 years. Baseline clinical characteristics of included patients were inconsistently reported across the studies. Data on cardiovascular comorbidities were available for 122 patients, while 105 and 89 patients had available data relating to antiplatelet and statin use, respectively. These data are summarised in table 2 . 

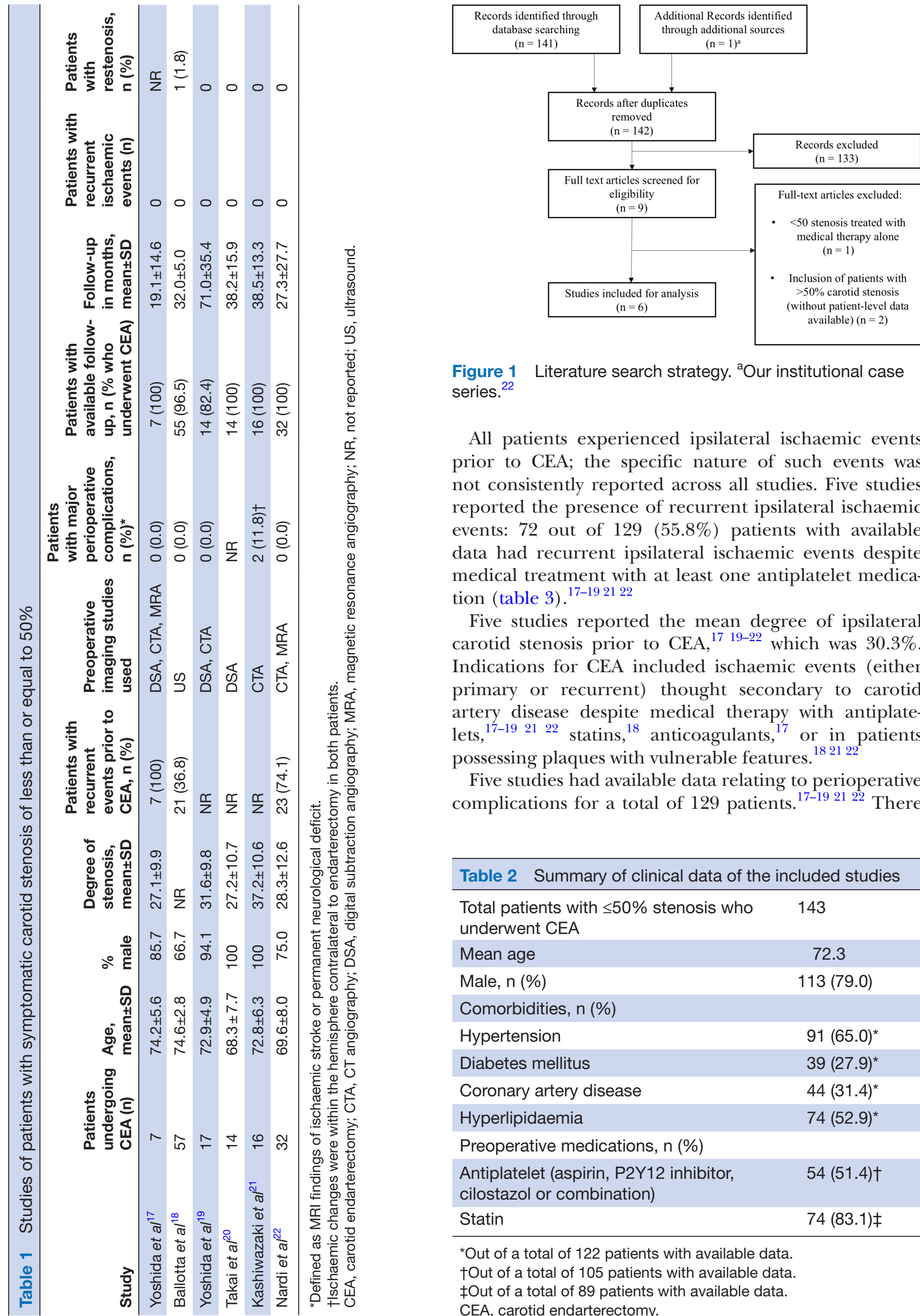

Figure 1 Literature search strategy. ${ }^{\text {aOO }}$ ur institutional case series. $^{22}$

All patients experienced ipsilateral ischaemic events prior to CEA; the specific nature of such events was not consistently reported across all studies. Five studies reported the presence of recurrent ipsilateral ischaemic events: 72 out of 129 (55.8\%) patients with available data had recurrent ipsilateral ischaemic events despite medical treatment with at least one antiplatelet medication (table 3). ${ }^{17-19} 2122$

Five studies reported the mean degree of ipsilateral carotid stenosis prior to CEA, ${ }^{17-22}$ which was $30.3 \%$. Indications for CEA included ischaemic events (either primary or recurrent) thought secondary to carotid artery disease despite medical therapy with antiplatelets, ${ }^{17-19} 2122$ statins, ${ }^{18}$ anticoagulants, ${ }^{17}$ or in patients possessing plaques with vulnerable features. ${ }^{182122}$

Five studies had available data relating to perioperative complications for a total of 129 patients. ${ }^{17-192122}$ There

\section{Table 2 Summary of clinical data of the included studies}

Total patients with $\leq 50 \%$ stenosis who 143 underwent CEA

\begin{tabular}{lc} 
Mean age & 72.3 \\
\hline Male, $\mathrm{n}(\%)$ & $113(79.0)$ \\
\hline $\begin{array}{l}\text { Comorbidities, } \mathrm{n}(\%) \\
\text { Hypertension }\end{array}$ & $91(65.0)^{\star}$ \\
\hline $\begin{array}{l}\text { Diabetes mellitus } \\
\text { Coronary artery disease }\end{array}$ & $39(27.9)^{\star}$ \\
\hline $\begin{array}{l}\text { Hyperlipidaemia } \\
\text { Preoperative medications, } \mathrm{n}(\%)\end{array}$ & $44(31.4)^{\star}$ \\
\hline $\begin{array}{l}\text { Antiplatelet (aspirin, P2Y12 inhibitor, } \\
\text { cilostazol or combination) }\end{array}$ & $74(52.9)^{\star}$ \\
\hline \begin{tabular}{l} 
Statin \\
\hline
\end{tabular} & $74(51.4) \dagger$ \\
\hline
\end{tabular}

*Out of a total of 122 patients with available data. tOut of a total of 105 patients with available data. ¥Out of a total of 89 patients with available data. CEA, carotid endarterectomy. 
Table 3 Recurrent ischaemic events prior to CEA in patients receiving medical therapy

\begin{tabular}{|c|c|c|}
\hline Study & $\begin{array}{l}\text { Patients with } \\
\text { recurrent ischaemic } \\
\text { events, } \mathrm{n} \text { (\% of total } \\
\text { patients undergoing } \\
\text { CEA) }\end{array}$ & $\begin{array}{l}\text { Medical therapy } \\
\text { prior to CEA }\end{array}$ \\
\hline Yoshida et al ${ }^{17}$ & $7(100)$ & $\begin{array}{l}\text { Dual antiplatelet } \\
\text { therapy in all } \\
\text { patients with } \\
\text { recurrent events*. }\end{array}$ \\
\hline Ballotta et $a l^{18}$ & $21(36.8)$ & $\begin{array}{l}\text { All with recurrent } \\
\text { events were } \\
\text { taking at least one } \\
\text { antiplatelet; } 13 \\
\text { were on a statin. }\end{array}$ \\
\hline Yoshida et al ${ }^{19}$ & $17(100)$ & $\begin{array}{l}\text { Dual antiplatelet } \\
\text { therapy in all } \\
\text { patients with } \\
\text { recurrent events. }\end{array}$ \\
\hline Takai et $a l^{20}$ & NR & - \\
\hline Kashiwazaki et a $2^{21}$ & $4(25.0)$ & $\begin{array}{l}\text { At least one } \\
\text { antiplatelet. }\end{array}$ \\
\hline Nardi et $a l^{22}$ & $23(71.9)$ & $\begin{array}{l}\text { At least one } \\
\text { antiplatelet. }\end{array}$ \\
\hline
\end{tabular}

*Antiplatelet medications consist of aspirin, P2Y12 inhibitor (ie, clopidogrel) and cilostazol.

CEA, carotid endarterectomy; NR, not reported.

were a total of nine patients $(6.9 \%)$ with perioperative complications. Seven patients $(5.4 \%)$ had minor perioperative complications: four patients had neck haematoma, two had transient cranial nerve palsies and one had bleeding from the incision site. Two patients $(1.6 \%)$ had major perioperative complications with positive diffusionweighted imaging MRI findings (both contralateral to the side of CEA); one patient also had transient hemiparesis that resolved completely in 30 days.

There were 138 patients with available long-term follow-up data. The weighted mean follow-up time was 36.1 months. There were no patients with recurrent ipsilateral ischaemic events following CEA. No cases of carotid restenosis were reported. Surgical and follow-up data for the included studies are found in table 4 .

Table 4 Surgical and follow-up data for patients who underwent CEA

\begin{tabular}{|c|c|}
\hline Mean degree of ipsilateral stenosis (\%) & 30.3 \\
\hline $\begin{array}{l}\text { Patients with major perioperative } \\
\text { complications, } \mathrm{n}(\%)\end{array}$ & $2 / 129(1.6)$ \\
\hline $\begin{array}{l}\text { Patients with available follow-up data, } n \text { (\% of } \\
\text { patients who underwent CEA) }\end{array}$ & $138(96.5)$ \\
\hline Mean follow-up, months & 36.1 \\
\hline Patients with recurrent ischaemic events, $\mathrm{n}$ & 0 \\
\hline
\end{tabular}

CEA, carotid endarterectomy.
Table 5 Summary data of patients with symptomatic carotid artery disease $\leq 50 \%$ stenosis with preoperative MRI findings of intraplaque haemorrhage

\begin{tabular}{ll}
\hline Patients, $\mathrm{n}$ & 28 \\
Male, $\mathrm{n}(\%)$ & $27(96.4)$ \\
\hline Mean age at time of CEA, years (SD) & $72.9(6.0)$ \\
\hline Mean degree of ipsilateral stenosis (SD) & $29.4(11.4)$ \\
$\begin{array}{l}\text { Patients with perioperative complications, } \\
\mathrm{n}(\%)\end{array}$ & $2(7.1)$ \\
\hline Follow-up, mean(SD) & $23.9(15.7)$ \\
\hline Patients with ischaemic events, $\mathrm{n}$ & 0 \\
\hline
\end{tabular}

CEA, carotid endarterectomy.

\section{Subgroup analysis of patients with IPH}

Twenty-eight patients from three studies ${ }^{17} 2122$ were identified to have IPH on MRI prior to CEA (table 5). Patient-level data were available in all cases. Twenty-seven (96.4\%) were male. The mean age at the time of CEA was $72.9 \pm 6.0$ years. The mean degree of ipsilateral carotid stenosis was $29.4 \% \pm 11.4$. There were two patients $(7.1 \%)$ with minor perioperative complications: one patient had neck swelling and one had transient hoarseness, both of which resolved completely at follow-up. The mean follow-up time following CEA was 23.9 \pm 15.7 months; no patients experienced recurrent ischaemic events or carotid restenosis.

\section{DISCUSSION}

In this study we reviewed a total of 143 patients from the published literature who underwent CEA for symptomatic carotid disease with $\leq 50 \%$ stenosis. Most of these patients $(55.8 \%)$ had recurrent ipsilateral cerebrovascular events despite medical management. We have found a low rate of major perioperative complications without any patients with available data demonstrating recurrent ipsilateral ischaemic events at follow-up. Similar findings were also observed for patients who underwent CEA for symptomatic carotid disease without stenosis who had MRI-based evidence of IPH on preoperative imaging. Based on these results, we hypothesise that CEA for symptomatic non-stenosing carotid atherosclerosis with highrisk features such as IPH might be beneficial in secondary stroke prevention.

Evidence is emerging that an important percentage of patients with the so-called ESUS may actually harbour vulnerable plaque features not readily apparent on conventional imaging studies. ${ }^{23}$ Intriguingly, Bogiatzi et $a l^{24}$ reported that defining large artery atherosclerosis by stenosis $>50 \%$, as used in ESUS studies, misses $79 \%$ of patients with large artery atherosclerosis defined by a carotid plaque burden in the top quartile $\left(>119 \mathrm{~mm}^{2}\right)$, with a $19.5 \%$ 5-year risk of stroke, myocardial infarction and/or vascular death. More recent classification systems have therefore defined large artery atherosclerosis by the degree of plaque burden as opposed to the per cent 
luminal stenosis. ${ }^{25}$ In turn, this classification system reclassifies some patients with ESUS as having large artery (ie, carotid artery) atherosclerosis.

In instances where the degree of luminal stenosis alone is used to classify patients as having ESUS, such patients with non-stenotic carotid disease may not be considered for surgical intervention based on established guidelines. ${ }^{4}$ For this specific reason, Goyal $e t a l^{11}$ hav e proposed a preliminary working definition of SyNC, which would imply that such patients would benefit from targeted therapy such as aggressive medical management or CEA. However, additional studies are needed in order to validate and refine the use of SyNC categorisation prior to widespread utilisation.

Given the lack of sufficient patient-level data within the included studies, SyNC definitions ${ }^{11}$ were unable to be applied. In particular, a definition of SyNC (either definite, probable or possible) requires the absence of acute or chronic ischaemic strokes in a vascular distribution other than the ipsilateral internal carotid artery (ICA); given that this information was unable to be obtained from the included studies, retroactive application of a SyNC diagnosis was precluded. However, the current data suggest that CEA in the context of minimal stenosis may be safe, well tolerated and potentially beneficial in terms of preventing stroke recurrence, which is an important first step in determining if CEA may be an effective targeted therapeutic in cases of SyNC. It is important to note, however, that without high-quality data relating to the annualised rate of recurrent strokes in patients with SyNC with intensive medical management (ie, randomised controlled trials including a CEA arm), it is not possible to draw definitive conclusions regarding the safety and efficacy of CEA in these patients.

Recent advances in imaging technology have led to increased emphasis on carotid plaque morphological features as markers of vulnerability as opposed to the degree of luminal stenosis alone. ${ }^{5}$ Several reports have indicated that recurrent ischaemic events may occur in the setting of vulnerable plaque features across the spectrum of luminal stenosis severity. ${ }^{91626} \mathrm{IPH}$, in particular, is now understood to be strongly associated with ipsilateral ischaemic events regardless of the degree of stenosis. ${ }^{16} 26$ We recently evaluated a cohort of 123 patients with ESUS at our institution who underwent carotid vessel wall imaging and found that IPH was present ipsilateral to the embolic event in $25.2 \%$ (31 of 123 ) of cases vs only $4.1 \%$ (5 of 123) contralaterally. ${ }^{27}$ Systematic reviews and metaanalyses of the literature have found that roughly $33 \%$ of patients with ESUS have plaque haemorrhage ipsilateral to their strokes. ${ }^{28}$ In our institutional cohort of over 120 patients with ESUS, approximately $30 \%$ of patients with ESUS and plaque haemorrhage suffered repeat ischaemic events despite medical management, necessitating CEA. In addition, a recent publication from the Carotid Plaque Imaging in Acute Stroke study revealed that the prevalence of complicated plaques (plaque surface rupture, luminal thrombosis and IPH) in patients with cryptogenic strokes was significantly higher ipsilateral $(31 \%)$ to the infarct compared with contralateral to the infarct $(12 \% ; \mathrm{p}<0.001) .{ }^{29}$ Specifically, the frequency of IPH in ipsilateral complicated carotid plaques was 28 of $32(88 \%)$ in patients with cryptogenic stroke. A metaanalysis of 9 studies with a total of 779 subjects by Gupta $e t$ $a l^{30}$ found that the presence of plaque haemorrhage was associated with an HR of 4.6 (95\% CI 2.91 to 7.24) for future ischaemic events and the annualised rate of stroke on medical therapy among patients with plaque haemorrhage was $17 \%$ per year.

These results suggest that plaque morphological features are important markers of plaque progression and instability and should therefore be taken into account when considering interventional measures, regardless of the degree of carotid luminal stenosis. However, the degree of carotid stenosis remains an important conventional feature of vulnerable plaque and, in the absence of more conclusive data, should continue to be a primary biomarker when considering CEA for symptomatic patients.

With advanced MRI-based sequences becoming more widely available, assessing patients for vulnerable plaque features including IPH will likely aid in identifying nonstenotic symptomatic plaques that will likely benefit from surgical intervention. The use of such advanced sequences including high-resolution vessel wall imaging is therefore advocated in order to more accurately select patients for interventional measures. In addition, novel high-resolution intravascular technologies promise to enable visualisation of the lumen/wall interphase at unprecedented resolution. ${ }^{78}$

The data from our study suggest that CEA in patients with symptomatic mild carotid stenosis may be beneficial in secondary stroke prevention, even in cases of highly unstable lesions harbouring IPH. However, the rate of recurrent strokes in patients with SyNC treated with intensive medical management is unknown, and the superiority of CEA in such cases relative to maximal medical therapy remains uncertain. In the current report, data regarding ischaemic recurrence rates prior to CEA were inconsistently reported across studies, although it is intriguing to note that all patients were free of recurrent ischaemic events at follow-up, even though several patients included had suffered multiple recurrent ischaemic events despite maximal medical therapy before being considered for CEA. This was similarly true in cases with IPH. These data might suggest that, in patients with symptomatic mild carotid artery stenosis (in particular, plaques with vulnerable features), CEA may be of value when added to medical management. Randomised studies are necessary to draw definitive conclusions.

\section{Limitations}

Our study is limited primarily as a result of the heterogeneity of studies included in the analysis; few 
variables were consistently reported across studies, therefore making a thorough report of all variables impossible. Specifically, the use of preoperative medications, including antiplatelets and statins, was incompletely reported across the included studies, which may play a confounding role in our data. Nevertheless, outcome variables (ie, perioperative and long-term follow-up results) were readily available, which enables some reasonable conclusions relating to safety and absence of long-term recurrent ischaemic events to be made. Although IPH is perhaps the most important plaque vulnerability feature recognised to date, other morphological features require attention, although these were not assessed across the included studies here due to incomplete reporting. Without a wellbalanced control group receiving intensive medical therapy for comparison, the superiority of CEA relative to medical management in the context of minimal carotid stenosis, although possible, remains uncertain.

\section{CONCLUSIONS}

CEA performed in the setting of symptomatic mild stenosis could be beneficial in terms of preventing recurrent ipsilateral ischaemic events and should be considered in highly selected cases. Additional studies of patients meeting the proposed criteria for SyNC are needed in order to evaluate the risk of ipsilateral recurrent stroke in patients with SyNC medically managed, define the imaging biomarker with the highest predictive risk for recurrent stroke and determine the added value of carotid revascularisation. Furthermore, subsequent studies with more robust methodology and advanced imaging are needed to draw more definitive conclusions regarding the efficacy and safety of endarterectomy for vulnerable plaques in the context of minimal stenosis relative to intensive medical therapy.

Contributors AL: data gathering, data analysis, draft writing, submission. VN, JB: data gathering, data analysis, draft editing. WB: concept design, data analysis, draft editing. GL, LS: concept design, data gathering, data analysis, draft editing.

Funding The authors have not declared a specific grant for this research from any funding agency in the public, commercial or not-for-profit sectors.

Competing interests None declared.

Patient consent for publication Not required.

Ethics approval All patients included in this study provided written informed consent for participation in research activities at our institution. This study was approved by the Mayo Clinic Institutional Review Board.

Provenance and peer review Not commissioned; externally peer reviewed. Data availability statement All data relevant to the study are included in the article or uploaded as supplemental information.

Open access This is an open access article distributed in accordance with the Creative Commons Attribution Non Commercial (CC BY-NC 4.0) license, which permits others to distribute, remix, adapt, build upon this work non-commercially, and license their derivative works on different terms, provided the original work is properly cited, appropriate credit is given, any changes made indicated, and the use is non-commercial. See: http://creativecommons.org/licenses/by-nc/4.0/.

\section{ORCID iD}

Anthony Larson http://orcid.org/0000-0001-6021-3452

\section{REFERENCES}

1 North American Symptomatic Carotid Endarterectomy Trial Collaborators, Barnett HJM, Taylor DW, et al. Beneficial effect of carotid endarterectomy in symptomatic patients with high-grade carotid stenosis. N Engl J Med 1991;325:445-53.

2 MRC European Carotid Surgery Trial: interim results for symptomatic patients with severe (70-99\%) or with mild (0-29\%) carotid stenosis. European Carotid Surgery Trialists' Collaborative Group. Lancet 1991;337:1235-43.

3 Ricotta JJ, Aburahma A, Ascher E, et al. Updated Society for vascular surgery guidelines for management of extracranial carotid disease: Executive summary. J Vasc Surg 2011;54:832-6.

4 Adams HP, Bendixen BH, Kappelle LJ, et al. Classification of subtype of acute ischemic stroke. definitions for use in a multicenter clinical trial. TOAST. Trial of ORG 10172 in acute stroke treatment. Stroke 1993;24:35-41.

5 Saba L, Yuan C, Hatsukami TS, et al. Carotid artery wall imaging: perspective and guidelines from the ASNR vessel wall imaging study Group and expert consensus recommendations of the American Society of Neuroradiology. AJNR Am J Neuroradiol 2018;39:E9-31.

6 Brinjikji W, Huston J, Rabinstein AA, et al. Contemporary carotid imaging: from degree of stenosis to plaque vulnerability. J Neurosurg 2016;124:27-42.

7 Savastano LE, Seibel EJ. Scanning fiber angioscopy: a multimodal intravascular imaging platform for carotid atherosclerosis. Neurosurgery 2017;64:188-98.

8 Savastano LE, Zhou Q, Smith A, et al. Multimodal laser-based angioscopy for structural, chemical and biological imaging of atherosclerosis. Nat Biomed Eng 2017;1. doi:10.1038/s41551-0160023. [Epub ahead of print: 1002 2017].

9 Freilinger TM, Schindler A, Schmidt C, et al. Prevalence of nonstenosing, complicated atherosclerotic plaques in cryptogenic stroke. JACC Cardiovasc Imaging 2012;5:397-405.

10 Wasserman BA, Wityk RJ, Trout HH, et al. Low-grade carotid stenosis: looking beyond the lumen with MRI. Stroke 2005;36:2504-13.

11 Goyal M, Singh N, Marko M, et al. Embolic stroke of undetermined source and symptomatic nonstenotic carotid disease. Stroke 2020;51:1321-5.

12 Moher D, Liberati A, Tetzlaff J, et al. Preferred reporting items for systematic reviews and meta-analyses: the PRISMA statement. PLOS Med 2009;6:e1000097.

13 Sterne JA, Hernán MA, Reeves BC, et al. ROBINS-I: a tool for assessing risk of bias in non-randomised studies of interventions. BMJ 2016;355:i4919.

14 Fritz VU, Levien LJ. Therapy for isolated, low and high grade symptomatic carotid artery stenosis. Ann Vasc Surg 1988;2:367-72.

15 Ahmed RM, Harris JP, Anderson CS, et al. Carotid endarterectomy for symptomatic, but "haemodynamically insignificant" carotid stenosis. Eur J Vasc Endovasc Surg 2010;40:475-82.

16 Altaf N, Daniels L, Morgan PS, et al. Detection of intraplaque hemorrhage by magnetic resonance imaging in symptomatic patients with mild to moderate carotid stenosis predicts recurrent neurological events. J Vasc Surg 2008;47:337-42.

17 Yoshida K, Sadamasa N, Narumi O, et al. Symptomatic low-grade carotid stenosis with intraplaque hemorrhage and expansive arterial remodeling is associated with a high relapse rate refractory to medical treatment. Neurosurgery 2012;70:1143-50. discussion 501.

18 Ballotta E, Angelini A, Mazzalai F, et al. Carotid endarterectomy for symptomatic low-grade carotid stenosis. J Vasc Surg 2014;59:25-31.

19 Yoshida K, Fukumitsu R, Kurosaki Y, et al. Carotid endarterectomy for medical therapy-resistant symptomatic low-grade stenosis. World Neurosurg 2019;123:e543-8.

20 Takai H, Uemura J, Yagita Y, et al. Plaque characteristics of patients with symptomatic mild carotid artery stenosis. J Stroke Cerebrovasc Dis 2018;27:1930-6.

21 Kashiwazaki D, Shiraishi K, Yamamoto S, et al. Efficacy of Carotid Endarterectomy for Mild (<50\%) Symptomatic Carotid Stenosis with Unstable Plaque. World Neurosurg 2019;121:e60-9.

22 Nardi V, Benson JC, Larson AS. Carotid artery endarterectomy in patients with symptomatic Non-Stenotic carotid artery disease. Stroke Vasc Neurol. forthcoming.

23 Singh N, Moody AR, Panzov V, et al. Carotid intraplaque hemorrhage in patients with embolic stroke of undetermined source. J Stroke Cerebrovasc Dis 2018;27:1956-9.

24 Bogiatzi C, Wannarong T, McLeod Al, et al. SPARKLE (subtypes of ischaemic stroke classification system), incorporating measurement of carotid plaque burden: a new validated tool for the classification of ischemic stroke subtypes. Neuroepidemiology 2014;42:243-51. 
25 Zhang H, Li Z, Dai Y, et al. Ischaemic stroke etiological classification system: the agreement analysis of CISS, SPARKLE and TOAST. Stroke Vasc Neurol 2019;4:123-8.

26 Altaf N, MacSweeney ST, Gladman J, et al. Carotid intraplaque hemorrhage predicts recurrent symptoms in patients with high-grade carotid stenosis. Stroke 2007;38:1633-5.

27 Larson AS, Nasr DM, Rizvi A, et al. Embolic stroke of undetermined source: the association with carotid intraplaque hemorrhage. JACC Cardiovasc Imaging 2021;14:506-8.
28 Kamtchum-Tatuene J, Wilman A, Saqqur M, et al. Carotid plaque with high-risk features in embolic stroke of undetermined source: systematic review and meta-analysis. Stroke 2020;51:311-4.

29 Kopczak A, Schindler A, Bayer-Karpinska A, et al. Complicated carotid artery plaques as a cause of cryptogenic stroke. J Am Coll Cardiol 2020;76:2212-22.

30 Gupta A, Baradaran H, Schweitzer AD, et al. Carotid plaque MR and stroke risk: a systematic review and meta-analysis. Stroke 2013;44:3071-7. 